\title{
Cartographies of Participation: How the changing natures of cartography has opened community and cartographer collaboration
}

\author{
William R Buckingham ' | wrbuckin@wisc.edu \\ Samuel F Dennis Jr. 1,2 | sfdennisji@wisc.edu
}

Nelson Institute for Environmental Studies, UW-Madison $550 \mathrm{~N}$ Park St

Madison, WI 53706
Department of Landscape Architecture, UW-Madison

1 Agricultural Hall, 1450 Linden Drive

Madison, WI 53706

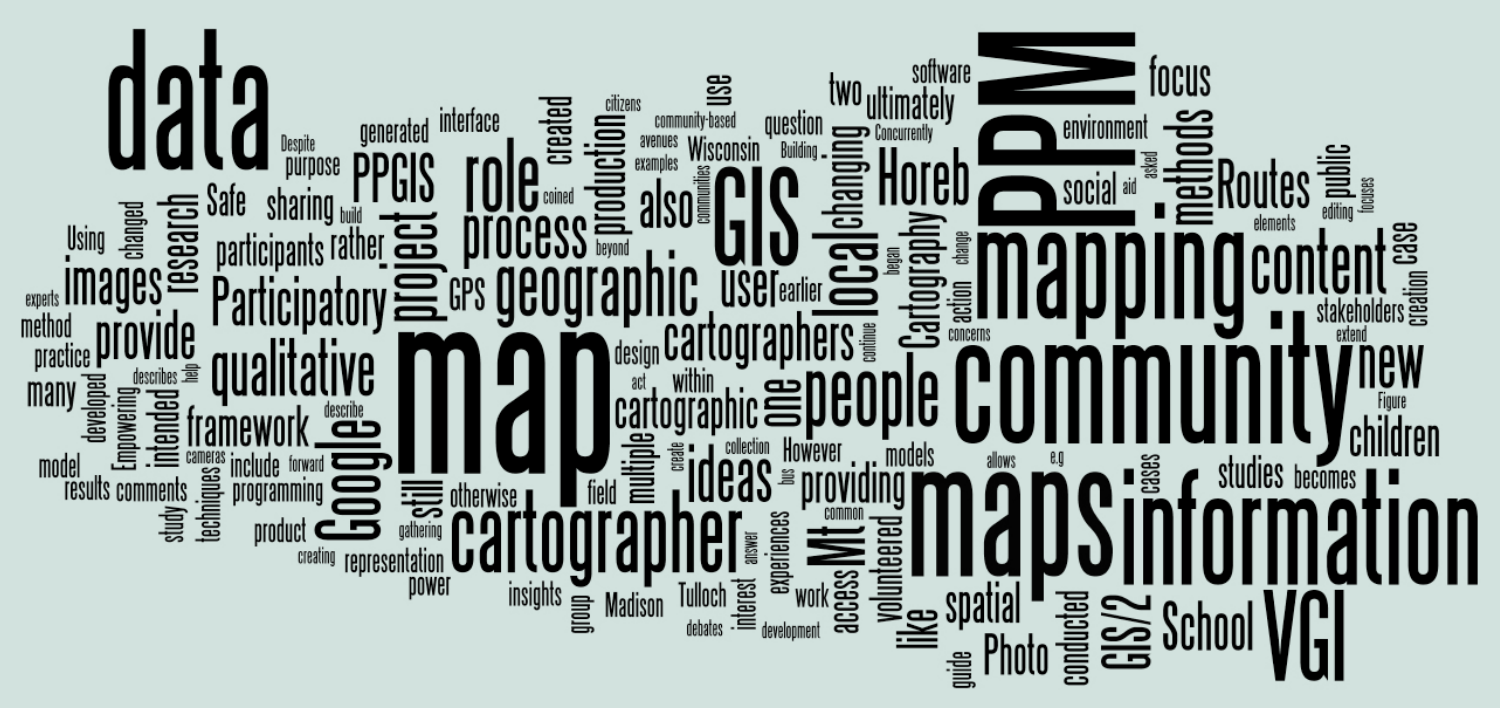

INTRODUCTION

Over the course of the past twenty years, a social awareness to maps and the practice of mapping has become a subject of acute interest both within the discipline of Geography and beyond. The rise of Google Maps, with its various "mashups" and "hacks" has produced an interest to use maps for understanding "non-mapped" phenomena (e.g. qualitative data or localized community information and knowledge). It is within this swirling of map interest that a potential avenue for Cartography can be seen - one that pushes the field forward while embracing the newfound enthusiasm of both the neo-geographer and the general public.

This special digital issue of $C P$ is intended to look at opportunities to share our craft. This sharing can come in many forms: a techniques piece, data or information. Our sharing focuses on inviting a group with specialized knowledge to participate in a mapping exercise. In situations like thissharing the practice of cartography - the cartographer plays the role of guide, more than map-maker, in the traditional sense of providing a finished product at the end of the production process. This role does not negate the 
cartographer but rather illustrates a model where the public's volunteered geographic information is both available and useful to expert and novice alike. Empowering the public to take certain courses of action based on the a newfound way of displaying spatial data in a way that they didn't know was possible.

In this paper we aim to illustrate the benefits of user-generated qualitative data and user participation and control of the mapping process. Specifically, we relate examples of how the role of the cartographer has changed from map creator - to map facilitator. We further describe two case studies illustrating new methods for implementing a "shared" cartography between experts and non-experts - methods that both aid community research and provide participants with the means to improve their community through the creation and use of maps. The case studies, in Madison and Mt. Horeb Wisconsin, focus on implementing web-based collection tools for the purpose of mapping the qualitative environment navigated by the residents.

\section{BACKGROUND}

Participatory mapping is a relatively nascent field, originating from debates between human geographers and GI scientists. From these debates a series of new research avenues have developed. These avenues overlap and build upon one another, creating a landscape where each subsequent development is not only rooted in the previous, but often takes only small steps away from the earlier models.

\section{Public Participation GIS (PPGIS):}

The oldest of the methods was the outcome of a gathering of social theorists and GIS practitioners, who developed a social critique of GIS, in Friday Harbor, Washington in 1993 (Sheppard 2005). Concurrently, GIS software became easier to use, removing the requirement of programming and providing a graphical user interface resembling more common software. This confluence of events opened up GIS for both wider adoption but also wider exposure to social and qualitative uses of GIS. However, Tulloch (2008) differentiates PPGIS from other methods as having a primary focus of providing access to data - not necessarily the creation of data by non-experts as in later models. From these beginnings, other emphases have evolved, (e.g., Neogeography, Volunteered Geographic Information, GIS/2).

\section{Volunteered Geographic Information (VGI):}

Goodchild (2007) coined the term VGI to describe this process of creation and dissemination of geographic data by individuals. Much like the new Internet ideas of Web 2.0 and user generated content; VGI offers the same opportunities for geographic information. The process produces results that provide unverified local scale information. However, without this process these results would otherwise go unnoticed. Tulloch (2008) focuses his description of this method on the collection of user created data, differentiating it from the PPGIS focus of access to data. Despite the differences, Tulloch goes on to state VGI is likely best served as a subset of PPGIS.

Volunteered Geographic Information (VGI) is the creation and dissemination of geographic data by individuals 
The speed with which changes are coming, however, requires that these fields adapt and mature quickly, or risk extinction.

\section{GIS/2:}

"A more equitable, accessible and empowering GIS" (Miller 2006), describes the closely related method of GIS/2. GIS/2 has similar origins to VGI; users can add data to overall datasets and provide insights that are missed otherwise. Miller points out that Google Maps has provided a more open format, which allows non-expert users to interact with the map, providing an early framework for a GIS/2. The constant evolution of new features of Google Maps, including things like My Maps which has now removed the programming barrier, has provided a technological framework for the implementation of both this model as well as later models, such as VGI.

We are in the midst of an exciting period of change, these developing specialty areas continue to reach more people, and will continue to empower communities with spatial representation of data. The following case studies focus on a two versions of these methods, both seeking to encourage localized representation of place.

\section{VGI IN PRACTICE - PARTICIPATORY PHOTO MAPPING AND SAFE ROUTES}

Before VGI was coined and while GIS/2 was (and still is) subject to multiple definitions, Stephen Matthews conducted a geo-ethnographic study that serves as a precursor to some of these ideas (Matthews et al. 2005). Matthews used GIS for non-numeric data and combined the resulting maps with ethnographic research to provide a detailed picture of the lives of the low-income families in his study. Using these ideas as a launching point, it becomes clear that qualitative data and maps can coexist and this has been illustrated in numerous cases (Dennis Jr 2006; Knigge and Cope 2006; Kwan and Ding 2008).

Building upon a desire to incorporate more qualitative techniques into a spatial framework, Participatory Photo Mapping (PPM) was created. With the goal of supporting young people as change agents, PPM leveraged ideas from community mapping, geo-ethnography and PPGIS (Dennis Jr et al. 2009). By extending these ideas to include visual images and narratives, the hope was to increase the power of the product - and ultimately to extend these analyses to a multi-methodological framework.

\section{PARTICIPATORY PHOTO MAPPING}

Participatory Photo Mapping (http://www.la.wisc.edu/ppm/) was developed to support community-based environmental assessment, action planning and policy development in the realm of health and place (Dennis Jr et al. 2008). PPM emerged from earlier work in community-based qualitative GIS (Dennis Jr 2006) and incorporates participatory practices from community mapping, photo elicitation and action research. Participants create photographs and narratives communicating their routine experience of their neighborhoods. Mapping these images and stories in a GIS helps communities plan actions to build a more health-supportive environment in which to live. 
PPM was created and implemented during the Youth Mapping for

Safe and Healthy Neighborhoods Initiative (Dennis Jr et al. 2009). The process involved groups of two to three young people, together with a member of the research team, equipped with a GPS receivers and cameras documenting the local environment. The children were asked to take pictures that communicated their experiences, especially places that held significant meaning for them. The images and the GPS tracks were then downloaded and imported into ArcGIS using the GPS PhotoLink plug-in, and the results were layered over an aerial photograph and presented to the children (Figure 1). During the presentation the entire group added their comments to photos. This process allowed multiple interpretations of places to be represented. For example, comments about the local park included descriptions ranging from "fun" to "scary."

Since this 2006 study, PPM has been utilized in a number of settings. The overarching theme from each PPM project has been the ability to expose the local experiences and concerns in each location. These local insights are often overlooked at all but the micro scale. In a community on the north side of Madison, Wisconsin, children on a PPM walk independently took multiple images of power lines just weeks after falling power lines had electrocuted two people waiting for the bus in a storm. These images revealed a fear shared by many local children that would have otherwise gone unnoticed. Similarly, many mobile basketball hoops were documented, representing settings for physical activity that do not exist on any published maps. These types of local experiences, coupled with a geographic representation, provide large-scale insights through a version of VGI.

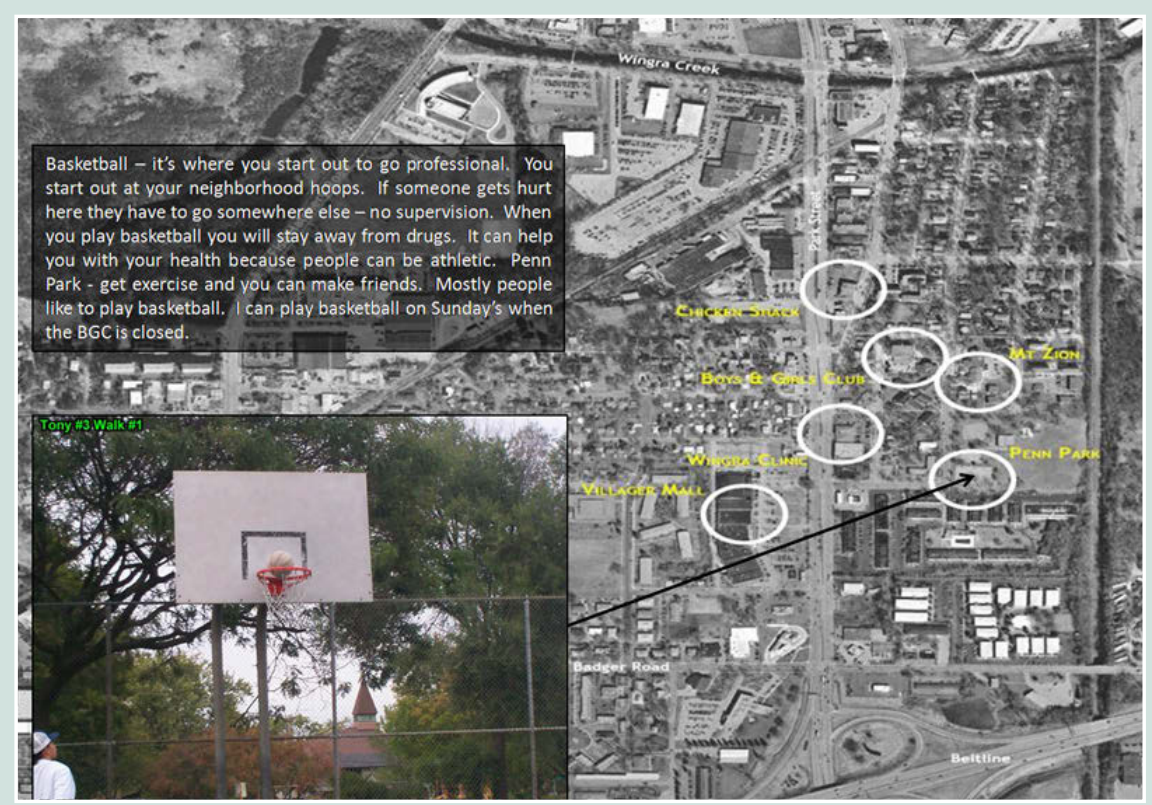

Figure 1 - Integration of Map, Photograph and Dialogue from PPM.

\section{MT HOREB}

Multiple PPM studies have been conducted since the initial PPM project was conducted on the south-side of Madison. Most of these have served 
the purpose of supporting people in small geographies, but none have extended the method - with one exception. The community of Mt. Horeb Wisconsin, a village of roughly 6,000 people, was the site of a PPM project in the spring of 2008. This project was an extension of Activate America's "On the Move" project conducted concurrently in Mt. Horeb. As a result of those partnerships it was suggested that PPM work be used in a Safe Routes to School effort - a PPM that would extend to include user generated and user controlled map content beyond photographs to include comments and edits left by the participants.

Residents of Mt. Horeb volunteered to lead "Walking School Buses" and were asked to draw their intended route on a large poster sized map. The Walking School Bus consisted of a parent volunteer leading children in a group walk to school. An overview of these routes appears in Figure 2. Once the routes were mapped they were digitized in a GIS and converted into KML. The KML was then loaded into Adobe Flex using the Google Maps API for Flash/Flex. The routes, PPM images and safety information (such as crosswalks with crossing guards, roundabouts and stoplights) were included in this interface. The route content for the map was generated by community and school members. After the mapping occurred discussions with community stakeholders began to discuss transferring ownership of the upload and editing of map content to members of the Mt. Horeb community.

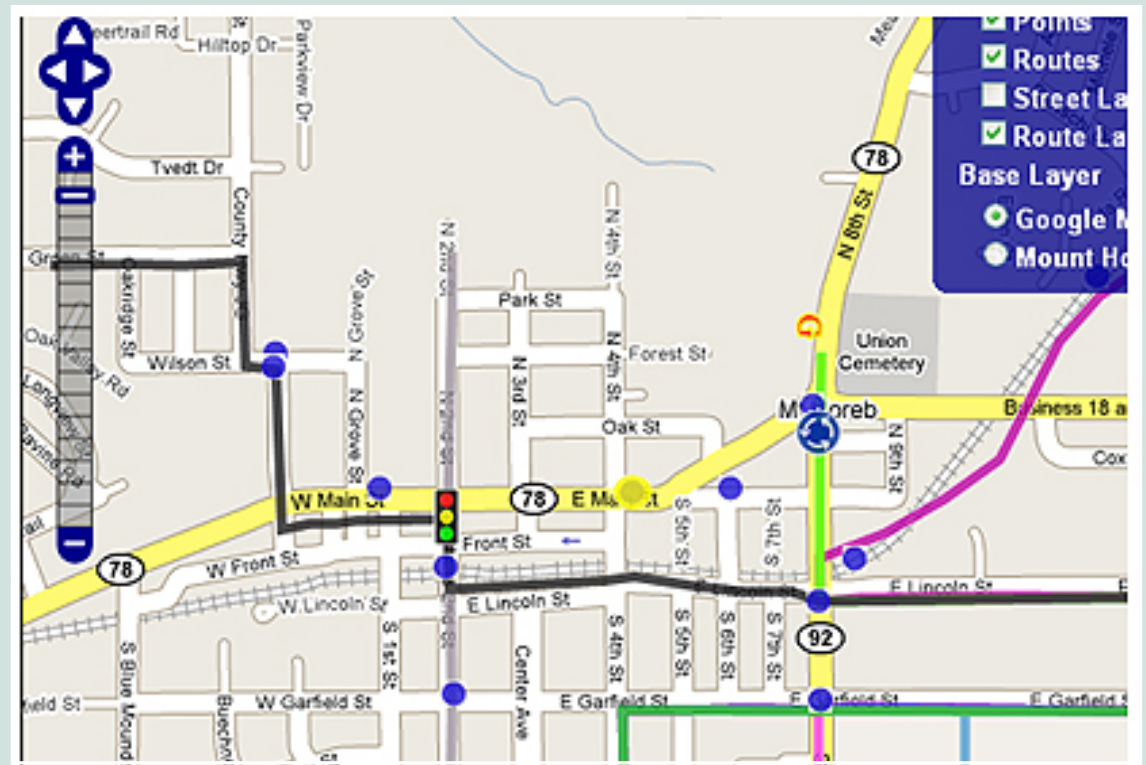

Figure 2 - Mt. Horeb Safe Routes Interactive Map with Walking School Bus Routes Encoded in Color.

During the efforts to determine who within the local community would maintain and manage the map content our team began to encounter resistance. Politics between the school district and the village made for a delicate balancing act. Concerns over liability and unfettered access to edit the map also created issues. Ultimately, the community stakeholders decided that the map should not provide open access for editing and update of content. Rather, the map would be moderated by volunteers and content 
would flow through the moderator to prevent a situation like Goodchild describes where "antisocial elements recognize and exploit vulnerabilities" by creating fictitious landscapes or adding defamatory information (Goodchild 2007). While not the optimal solution, having community stakeholders act as the moderators, the community still owns the map, and controls map updates.

This shift to community ownership of both the map and its geographic information illustrates the changing role of the cartographer. In most PPM cases to date, the cartographic product was still produced by experts, more or less in situations resembling common cartographic production. The Mt. Horeb case changed the role of the cartographer from one of final map production as in the earlier PPM cases, to one of map production for the purpose of data gathering at the beginning of the project, both by including Google base maps, but also by placing data production and update on the community participants. Here, the cartographer becomes more of a guide, building a template for the look and feel of data, representing the initial interface and providing support and training. Almost in the role of a software developer - but with a specific focus on the map and the layout of the tools, the cartographer is no less important than in the PPM examples - rather the role has just changed.

\section{TAKE - AWAYS}

Both PPM and extensions of PPM like the Safe Routes project highlight the needs for cartographic expertise, but also the changing role that cartographers are playing. Despite leveraging Google Maps, the design of the map elements still requires the same cartographic design principles to aid in understanding. The cartographer in this model is no longer purely designing for communication of ideas, but rather is truly aiming for the sharing of information and ideas between parties - particularly between citizens, decision makers and researchers.

\section{CHANGING TECHNOLOGY}

The continuing diversification of cartography leads to some challenges when it comes to defining the field. Changing technologies (such as Google Maps) as well as new branches of GIScience (such as VGI) require cartographers to remain nimble and ready to evolve. Ultimately the question becomes who is a cartographer? Is it Google hackers, people trained in design and projections, computer scientists, ordinary citizens, or is it a mix of all of these depending on both the intended map output but also the intended utility of the map. This is the unanswerable question - the definition is changing so rapidly, between GPS enable cameras to new and diverse programming languages, that the answer is difficult to pin down. Ultimately, we may find that there is no one good answer to this question. The era of the cartographer deciding what data should appear on a map and remaining the authority over the map has ended-geographic information is in the wild and cartographers need to jump on board to help guide the revolution. 


\section{CONCLUSION}

PPGIS, VGI, PPM are methods and techniques that are all part of the transition of many things: GIS, public consumption of spatial information, the content and output cartographers create, and the role of cartographers. This milieu of uncertainty however doesn't alter the fact that sound cartographic training, mixed with a flexible framework, allows for a solid grounding going boldly forward into a new world of spatial information. Cartographers and cartography are still necessary for the work they have long performed, but they are also critical in empowering people to make their own maps. These maps have the potential to give a voice to the unspoken, a home to the unmapped, and most importantly to help people use and understand maps to make decisions.

\section{BIBLIOGRAPHY}

Dennis Jr, S. F. (2006). "Prospects for qualitative GIS at the intersection of youth development and participatory urban planning." Environment and Planning A 38: 2039-2054.

Dennis Jr, S. F., S. Gaulocher, R. M. Carpiano and D. Brown (2009). “Participatory photo mapping (PPM): Exploring an integrated method for health and place research with young people." Health and Place 15(2): 466-473.

Goodchild, M. F. (2007). "Citizens as sensors: the world of volunteered geography." GeoJournal 69: 211-221.

Goodchild, M. F. (2008). “Commentary: wither VGI?” GeoJournal 72: 239-244.

Knigge, L. and M. Cope (2006). “Grounded visualization: integrating the analysis of qualitative and quantitative data through grounded theory and visualization." Environment and Planning A 38: 2021-2037.

Kwan, M.-P. and G. Ding (2008). "Geo-Narrative: Extending Geographic Information Systems for Narrative Analysis in Qualitative and Mixed-Methods Research.” The Professional Geographer 60(4): 443-465.

Matthews, S. A., J. E. Detwiler and L. M. Burton (2005). “Geo-ethnography: coupling Geographic Information Analysis Techniques with Ethnographic Methods in Urban Research.” Cartographica 40(4): 75-90.

Miller, C. C. (2006). "A Beast in the Field: The Google Maps Mashup as GIS/2." Cartographica 41(3): 187-199.

Sheppard, E. (2005). "Knowledge Productions through Critical GIS: Genealogy and Prospects.” Cartographica 40(4): 5-21.

Sieber, R. (2006). "Public Participation Geographic Information Systems: A Literature Review and Framework." Annals of the Association of American Geographers 96(3): 491-507.

Tulloch, D. L. (2008). "Is VGI participation? From vernal pools to video games.” GeoJournal 72: 161-171. 